PROCEEDINGS OF THE

AMERICAN MATHEMATICAL SOCIETY

Volume 139, Number 12, December 2011, Pages 4497-4511

S 0002-9939(2011)10860-8

Article electronically published on April 25, 2011

\title{
ON MAXIMAL RANGES OF VECTOR MEASURES FOR SUBSETS AND PURIFICATION OF TRANSITION PROBABILITIES
}

\author{
PENG DAI AND EUGENE A. FEINBERG
}

(Communicated by Richard C. Bradley)

\begin{abstract}
Consider a measurable space with an atomless finite vector measure. This measure defines a mapping of the $\sigma$-field into a Euclidean space. According to the Lyapunov convexity theorem, the range of this mapping is a convex compactum. Similar ranges are also defined for measurable subsets of the space. Two subsets with the same vector measure may have different ranges. We investigate the question whether, among all the subsets having the same given vector measure, there always exists a set with the maximal range of the vector measure. The answer to this question is positive for two-dimensional vector measures and negative for higher dimensions. We use the existence of maximal ranges to strengthen the Dvoretzky-Wald-Wolfowitz purification theorem for the case of two measures.
\end{abstract}

\section{INTRODUCTION}

Let $(X, \mathcal{F})$ be a measurable space and $\mu=\left(\mu_{1}, \ldots, \mu_{m}\right), m=1,2, \ldots$, be a finite atomless vector measure on it. We recall that a measure $\nu$ is called atomless if for each $Z \in \mathcal{F}$, such that $\nu(Z)>0$, there exists $Z^{\prime} \in \mathcal{F}$ such that $Z^{\prime} \subset Z$ and $0<\nu\left(Z^{\prime}\right)<\nu(Z)$. A vector measure $\mu=\left(\mu_{1}, \ldots, \mu_{m}\right)$, is called finite and atomless if each measure $\mu_{i}, i=1, \ldots, m$, is finite and atomless. For each $Y \in \mathcal{F}$ consider the range $R_{\mu}(Y)=\{\mu(Z): Z \in \mathcal{F}, Z \subseteq Y\}$ of the vector measures of all its measurable subsets $Z$. According to the Lyapunov convexity theorem $\left[\right.$, the sets $R_{\mu}(Y)$ are convex compactums in $\mathbb{R}^{m}$. For a review on this theorem and its applications, see [9].

In this paper we study whether for any $p \in R_{\mu}(X)$, the set of all ranges $\left\{R_{\mu}(Y)\right.$ : $\mu(Y)=p, Y \in \mathcal{F}\}$ contains a maximal element. In other words, is it always true that for any $p \in R_{\mu}(X)$ there exists a subset $Y^{*} \in \mathcal{F}$ such that $\mu\left(Y^{*}\right)=p$ and $R_{\mu}\left(Y^{*}\right) \supseteq R_{\mu}(Y)$ for any $Y \in \mathcal{F}$ with $\mu(Y)=p$ ? We show that the answer is positive when $m=2$ and negative when $m>2$. Furthermore, for $m=2$, this maximal range can be constructed by a simple geometric transformation of $R_{\mu}(X)$.

In addition to the maximal range, it is possible to consider a minimal range. For $q \in R_{\mu}(X)$, the set $M^{*} \in \mathcal{F}$ with $\mu\left(M^{*}\right)=q$ has minimal range corresponding to

Received by the editors June 1, 2010 and, in revised form, June 16, 2010 and October 20, 2010. 2010 Mathematics Subject Classification. Primary 60A10, 28A10.

Key words and phrases. Lyapunov convexity theorem, maximal subset, purification of transition probabilities.

This research was partially supported by NSF grants CMMI-0900206 and CMMI-0928490.

(c)2011 American Mathematical Society Reverts to public domain 28 years from publication 
$q$ if $R_{\mu}(M) \supseteq R_{\mu}\left(M^{*}\right)$ for any $M \in \mathcal{F}$ with $\mu(M)=q$. We show that a set has a maximal range corresponding to $p$ if and only if its complement has a minimal range corresponding to $\mu(X)-p$. Therefore, minimal ranges also exist for dimension two and they may not exist for higher dimensions.

Lyapunov's theorem is relevant to the purification of transition probabilities discovered by Dvoretzky, Wald and Wolfowitz [2, 3] for a finite image set. Let $(A, \mathcal{A})$ be a measurable space and $\pi$ be a transition probability from $X$ to $A$; that is, $\pi(B \mid x)$ is a measurable function on $(X, \mathcal{F})$ for any $B \in \mathcal{A}$ and $\pi(\cdot \mid x)$ is a probability measure on $(A, \mathcal{A})$ for any $x \in X$. According to Dvoretzky, Wald and Wolfowitz [2, 3, two transition probabilities $\pi_{1}$ and $\pi_{2}$ are called strongly equivalent if

$$
\int_{X} \pi_{1}(B \mid x) \mu_{i}(d x)=\int_{X} \pi_{2}(B \mid x) \mu_{i}(d x), \quad i=1, \ldots, m, \quad B \in \mathcal{A} .
$$

A transition probability $\pi$ is called pure if each measure $\pi(\cdot \mid x)$ is concentrated at one point. A pure transition probability $\pi$ is defined by a measurable mapping $\varphi: X \rightarrow A$ such that $\pi(B \mid x)=I\{\varphi(x) \in B\}$ for all $B \in \mathcal{A}$. According to the contemporary terminology, a transition probability can be purified if for it there exists a strongly equivalent pure transition probability.

For a finite set $A$, Dvoretzky, Wald and Wolfowitz [2, 3 proved that any transition probability can be purified (we recall that $\mu$ is atomless). Edwards 4, Theorem 4.5] generalized this result to the case of a countable set $A$. Khan and Rath [6. Theorem 2] gave another proof of this generalization. Loeb and Sun [7, Example 2.7] constructed an elegant example when a transition probability cannot be purified for $m=2, X=[0,1]$, and $A=[-1,1]$. However, purification holds for a countable set of nonatomic, finite, signed Loeb measures when $A$ is a complete separable metric space [7, Corollary 2.6].

Note that for a countable (finite or infinite) set $A$, a transition probability $\pi$ can be purified if and only if there exists a partition $\left\{Z^{a} \in \mathcal{F}: a \in A\right\}$ of $X$ such that

$$
\int_{X} \pi(a \mid x) \mu(d x)=\mu\left(Z^{a}\right), \quad a \in A,
$$

where $\mu$ is an $m$-dimensional finite atomless vector measure. Since $\int_{X} \pi(a \mid x) \mu(d x)$ are vectors in $\mathbb{R}^{m}$, a natural question is: under what conditions for an arbitrary set of vectors $\left\{p^{a}: a \in A\right\}$ does there exist a partition $\left\{Z^{a} \in \mathcal{F}: a \in A\right\}$ of $X$ such that $p^{a}=\mu\left(Z^{a}\right)$ for each $a \in A$ ? We use the theorem on maximal ranges proved in this paper to show that for $m=2$, such a partition exists if and only if (i) $\sum_{a \in A} p^{a}=\mu(X)$, and (ii) $\sum_{a \in B} p^{a} \in R_{\mu}(X)$ for any finite subset $B$ of $A$. For $m=2$, the Dvoretzky-Wald-Wolfowitz theorem for a countable set $A$ [4, 6] follows from the sufficient part of this statement.

We formulate the main results in the following section, prove the existence of maximal and minimal subsets for $m=2$ in Section 3, provide counterexamples when $m>2$ in Section 4, and describe geometric constructions of maximal ranges in Section 5. Section 6 is devoted to the proof of the theorem on the existence of a partition.

\section{MAin Results}

Definition 2.1. Given a measurable subset $Y$ of the measurable space $(X, \mathcal{F})$ with a vector measure $\mu$ and a vector $p \in R_{\mu}(Y)$, we define 
(a) the set of all subsets of $Y$ with vector measure $p$,

$$
\mathcal{S}_{\mu}^{p}(Y)=\left\{Z \in \mathcal{F}_{Y}: \mu(Z)=p\right\},
$$

where $\mathcal{F}_{Y}=\{Z \subseteq Y: Z \in \mathcal{F}\}$;

(b) the union of all the ranges of all subsets of $Y$ with the vector measure $p$,

$$
R_{\mu}^{p}(Y)=\bigcup_{Z \in \mathcal{S}_{\mu}^{p}(Y)} R_{\mu}(Z) ;
$$

(c) the intersection of the $R_{\mu}(Y)$ with its shift by a vector $-(\mu(Y)-p)$,

$$
Q_{\mu}^{p}(Y)=\left(R_{\mu}(Y)-\{\mu(Y)-p\}\right) \cap R_{\mu}(Y),
$$

where $S_{1}-S_{2}=\left\{q-r: q \in S_{1}, r \in S_{2}\right\}$ for $S_{1}, S_{2} \in \mathbb{R}^{m}$. In particular, $R_{\mu}(Y)-\{r\}$ is a parallel shift of $R_{\mu}(Y)$ by $-r$.

Definition 2.2. For a measurable subset $Y \in \mathcal{F}$, the set $Z^{*} \in \mathcal{S}_{\mu}^{p}(Y)$ such that $R_{\mu}\left(Z^{*}\right)=R_{\mu}^{p}(Y)$ is called the maximal subset of $Y$ with the measure $p$. The set $M^{*} \in \mathcal{S}_{\mu}^{q}(Y)$ such that $R_{\mu}\left(M^{*}\right) \subseteq R_{\mu}(M)$ for any $M \in \mathcal{S}_{\mu}^{q}(Y)$ is called the minimal subset of $Y$ with the measure $q$.

Our first result is the following theorem.

Theorem 2.3. For a two-dimensional finite atomless vector measure $\mu=\left(\mu_{1}, \mu_{2}\right)$ and for a vector $p \in R_{\mu}(X)$, there exists a maximal set $Z^{*} \in \mathcal{S}_{\mu}^{p}(X)$ and, in addition, $R_{\mu}^{p}(X)=Q_{\mu}^{p}(X)$.

Theorem 2.3 immediately implies that the set $R_{\mu}^{p}(X)$, which is the union of the ranges of $\mu$ on $Z$, for all $Z \in \mathcal{S}_{\mu}^{p}(X)$, is a convex compactum. Furthermore, if $R_{\mu}(X)$ and $p$ are given, the set $R_{\mu}^{p}(X)$ is defined by two simple geometric operations, a shift and an intersection, since $Q_{\mu}^{p}(X)$ is defined by these operations.

The following theorem links the notions of maximal and minimal subsets.

Theorem 2.4. The set $Z^{*}$ is the maximal subset of $X$ with the measure $p$ if and only if $M^{*}=X \backslash Z^{*}$ is the minimal subset of $X$ with the measure $\mu(X)-p$.

We will use Theorem 2.3 to prove the following theorem that, as shown in Section 6. strengthens the Dvoretzky-Wald-Wolfowitz purification theorem [4, 6] for the case $m=2$.

Theorem 2.5. Consider a measurable space $(X, \mathcal{F})$ with a two-dimensional finite atomless vector measure $\mu$, a countable set $A$, and a set of two-dimensional vectors $\left\{p^{a}: a \in A\right\}$. A partition $\left\{Z^{a} \in \mathcal{F}: a \in A\right\}$ of $X$, with $p^{a}=\mu\left(Z^{a}\right)$ for all $a \in A$, exists if and only if (i) $\sum_{a \in A} p^{a}=\mu(X)$ and (ii) $\sum_{a \in B} p^{a} \in R_{\mu}(X)$ for any finite subset $B \subset A$.

\section{Maximal And minimal subsets}

In this section, we prove Theorems 2.3 and 2.4. Recall that for a set $S \subseteq \mathbb{R}^{m}$, its reflection across a point $c \in \mathbb{R}^{m}$ is $\operatorname{Ref}(S, c)=\{2 c\}-S$. If $S=\{s\}$ is a singleton, we shall write $\operatorname{Ref}(s, c)$ instead of $\operatorname{Ref}(\{s\}, c)$. A set $S \subseteq \mathbb{R}^{m}$ is called centrally symmetric if $\operatorname{Ref}(S, c)=S$ for some point $c \in \mathbb{R}^{m}$ called the center of $S$. Any bounded centrally symmetric set has only one center.

In this section we let $Y \in \mathcal{F}$ be any measurable subset of $X$. Lemmas 3.1 3.3 hold for any finite atomless vector measure $\mu=\left(\mu_{1}, \ldots, \mu_{m}\right)$ on $(X, \mathcal{F})$, where $m=1,2, \ldots$. 
Lemma 3.1. The set $R_{\mu}(Y)$ is centrally symmetric with the center $\frac{1}{2} \mu(Y)$.

Proof. The proof is straightforward, and this fact was observed by Lyapunov 8 , p. 476].

Lemma 3.2. The equality $R_{\mu}(Y)-\{\mu(Y)-p\}=\operatorname{Ref}\left(R_{\mu}(Y), \frac{1}{2} p\right)$ holds for any $p \in R_{\mu}(Y)$.

Proof. By Lemma 3.1, $R_{\mu}(Y)=\operatorname{Ref}\left(R_{\mu}(Y), \frac{1}{2} \mu(Y)\right)=\{\mu(Y)\}-R_{\mu}(Y)$. Therefore, $R_{\mu}(Y)-\{\mu(Y)-p\}=\left(\{\mu(Y)\}-R_{\mu}(Y)\right)-\{\mu(Y)-p\}=\{p\}-R_{\mu}(Y)=$ $\operatorname{Ref}\left(R_{\mu}(Y), \frac{1}{2} p\right)$.

Lemma 3.3. Each of the sets $R_{\mu}^{p}(Y)$ and $Q_{\mu}^{p}(Y)$ is centrally symmetric with the center $\frac{1}{2} p$.

Proof. According to Lemma 3.1 each set $Z \in \mathcal{S}_{\mu}^{p}(Y)$ is centrally symmetric with the center $\frac{1}{2} p$. Therefore, $R_{\mu}^{p}(Y)$, which is the union of all the sets in $Z \in \mathcal{S}_{\mu}^{p}(Y)$, is also centrally symmetric with the center $\frac{1}{2} p$.

In addition,

$$
\begin{aligned}
\operatorname{Ref}\left(Q_{\mu}^{p}(Y), \frac{1}{2} p\right) & =\operatorname{Ref}\left(\left(R_{\mu}(Y)-\{\mu(Y)-p\}\right) \cap R_{\mu}(Y), \frac{1}{2} p\right) \\
& =\operatorname{Ref}\left(\operatorname{Ref}\left(R_{\mu}(Y), \frac{1}{2} p\right) \cap R_{\mu}(Y), \frac{1}{2} p\right) \\
& =R_{\mu}(Y) \cap \operatorname{Ref}\left(R_{\mu}(Y), \frac{1}{2} p\right) \\
& =R_{\mu}(Y) \cap\left(R_{\mu}(Y)-\{\mu(Y)-p\}\right)=Q_{\mu}^{p}(Y),
\end{aligned}
$$

where the first and last equalities follow from the definition of $Q_{\mu}^{p}$, and the second and second to last equalities follow from Lemma 3.2. The third equality holds because a reflection of intersections equals the intersection of reflections and, in addition, a reflection of a reflection across the same point is the original set.

Here we present the major ideas of the proof of Theorem 2.3. First, as shown later, after Theorem 2.3 is proven for equivalent measures $\mu_{1}$ and $\mu_{2}$, this condition can be removed. So, we make the following assumption in Lemmas 3.5, 3.7,3.13,

Assumption 3.4. The measures $\mu_{1}$ and $\mu_{2}$ are finite, atomless, and equivalent.

Under Assumption 3.4 let $f(x)=\frac{d \mu_{2}}{d \mu_{1}}(x)$ be a Radon-Nikodým derivative of $\mu_{2}$ with respect to $\mu_{1}$. Since $f$ is defined $\mu_{1}$-a.e., we fix any of its versions. We shall frequently use notation similar to

$$
\{f(x)<l\}=\{x \in X: f(x)<l\} .
$$

Second, under Assumption 3.4, for any $a \in\left[0, \mu_{1}(X)\right]$, we denote

$$
l_{a}=\min \left\{l \geq 0: \mu_{1}(\{f(x) \leq l\}) \geq a\right\} .
$$

Observe that the minimum in (3.1) exists. Indeed, let

$$
l_{a}=\inf \left\{l \geq 0: \mu_{1}(\{f(x) \leq l\}) \geq a\right\} .
$$

We need to show that $\mu_{1}\left(\left\{f(x) \leq l_{a}\right\}\right) \geq a$. If $l_{a}=\infty$, then $\mu_{1}(\{f(x) \leq \infty\})=$ $\mu_{1}(X) \geq a$. Let $l_{a}<\infty$. Consider a sequence $l^{k} \searrow l_{a}, k=1,2, \ldots$. Then 
$\bigcap_{k=1}^{\infty}\left\{f(x) \leq l^{k}\right\}=\left\{f(x) \leq l_{a}\right\}$ and $\left\{f(x) \leq l^{k}\right\} \supseteq\left\{f(x) \leq l^{k+1}\right\}$. Therefore $\mu_{1}\left(\left\{f(x) \leq l_{a}\right\}\right)=\lim _{k \rightarrow \infty} \mu_{1}\left(\left\{f(x) \leq l^{k}\right\}\right) \geq a$.

Third, it is possible to construct the maximal set $Z^{*}=X \backslash M^{*}$, where $M^{*}$ can be defined explicitly. Let $X^{l}=\{f(x)=l\}$. If $\mu_{1}\left(X^{l}\right)=0$ for all $l \in[0, \infty)$, then the definition of $M^{*}$ is easier and we explain it first. In this case, there exists $a^{*} \in\left[0, \mu_{1}(X)\right]$ such that $\mu_{2}\left(M^{*}\right)=\mu_{2}(X)-p_{2}$, and $M^{*}$ can be defined as

$$
M^{*}=\left\{l_{a^{*}} \leq y<l_{a^{*}+\left(\mu_{1}(X)-p_{1}\right)}\right\} .
$$

In the general situation, the number $a^{*}$ can be chosen to satisfy

$$
\mu_{2}\left(\left\{l_{a^{*}}<y<l_{a^{*}+\left(\mu_{1}(X)-p_{1}\right)}\right\}\right) \leq \mu_{2}(X)-p_{2} \leq \mu_{2}\left(\left\{l_{a^{*}} \leq y \leq l_{a^{*}+\left(\mu_{1}(X)-p_{1}\right)}\right\}\right)
$$

and

$$
M^{*}=\left\{l_{a^{*}}<y<l_{a^{*}+\left(\mu_{1}(X)-p_{1}\right)}\right\} \cup Z^{1} \cup Z^{2},
$$

for $Z^{i}, i=1,2$, being some measurable subsets of $X^{l^{i}}$, where $l^{1}=l_{a^{*}}$ and $l^{2}=$ $l_{a^{*}+\left(\mu_{1}(X)-p_{1}\right)}$. In particular, if $\mu_{1}\left(X^{l^{1}}\right)=0$, let $Z^{1}=X^{l^{1}}$, and if $\mu_{1}\left(X^{l^{2}}\right)=0$, let $Z^{2}=\emptyset$. If $\mu_{1}\left(X^{l^{1}}\right)=\mu_{1}\left(X^{l^{2}}\right)=0$, then (3.3) reduces to (3.2). It is easy to show that the number of $l$ such that $\mu_{1}\left(X^{l}\right)=0$ is countable, but we do not use this fact.

The proof of Theorem 2.3 is based on several lemmas.

Lemma 3.5. Under Assumption [3.4, the numbers $l_{a}, a \in\left[0, \mu_{1}(X)\right]$ have the following properties: (a) $\mu_{1}\left(\left\{f(x)<l_{a}\right\}\right) \leq a \leq \mu_{1}\left(\left\{f(x) \leq l_{a}\right\}\right)$; (b) $l_{a} \leq l_{a^{\prime}}$ if $a \leq a^{\prime}$.

Proof. For (a), by definition, $a \leq \mu_{1}\left(\left\{f(x) \leq l_{a}\right\}\right)$. To prove that $\mu_{1}\left(\left\{f(x)<l_{a}\right\}\right)$ $\leq a$, assume that $\mu_{1}\left(\left\{f(x)<l_{a}\right\}\right)>a$. If $l_{a}=0$, then $\mu_{1}\left(\left\{f(x)<l_{a}\right\}\right)=0>a$, which contradicts the assumption that $a \geq 0$. If $l_{a}>0$, let $\epsilon_{k} \searrow 0, k=1,2, \ldots$, be a sequence of positive numbers. Then, for $k=1,2, \ldots$,

$$
\mu_{1}\left(\left\{f(x)<l_{a}\right\}\right)=\mu_{1}\left(\left\{f(x) \leq l_{a}-\epsilon_{k}\right\}\right)+\mu_{1}\left(\left\{l_{a}-\epsilon_{k}<f(x)<l_{a}\right\}\right)>a .
$$

Let $D_{k}=\left\{l_{a}-\epsilon_{k}<f(x)<l_{a}\right\}$. We observe that $D_{k+1} \subseteq D_{k}$ and $\bigcap_{u=1}^{\infty} D_{k}=\emptyset$. Therefore, $\lim _{k \rightarrow \infty} \mu_{1}\left(D_{k}\right)=0$. Thus, $\mu_{1}\left(f(x) \leq l_{a}-\epsilon\right)>a$ for some $\epsilon>0$ and this contradicts (3.1). These contradictions imply the lemma.

For (b), assume $l_{a}>l_{a^{\prime}}$. Then $\mu_{1}\left(\left\{f(x) \leq l_{a^{\prime}}\right\}\right) \geq a^{\prime} \geq a$, and this contradicts (3.1).

Note that for each $l \in[0, \infty)$, there exists a subfamily

$$
\left\{W_{b}\left(X^{l}\right) \in \mathcal{F}_{X^{l}}: b \in\left[0, \mu_{1}\left(X^{l}\right)\right]\right\}
$$

such that $W_{b}\left(X^{l}\right) \subset W_{b^{\prime}}\left(X^{l}\right) \subseteq X^{l}$ whenever $b<b^{\prime} \leq \mu_{1}\left(X^{l}\right)$ and $\mu_{1}\left(W_{b}\left(X^{l}\right)\right)$ $=b$ for each $b \in\left[0, \mu_{1}\left(X^{l}\right)\right]$. This fact follows from Ross [10, Theorem 2(LT3)]. We set $W_{0}\left(X^{l}\right)=\emptyset$. From now on we fix a family of $W_{b}\left(X^{l}\right)$ for each $l \in[0, \infty)$.

Definition 3.6. Under Assumption 3.4 for each $a$, define the following set:

$$
L_{a}=\left\{f(x)<l_{a}\right\} \cup W_{c}\left(X^{l_{a}}\right),
$$

where $c=a-\mu_{1}\left(\left\{f(x)<l_{a}\right\}\right)$.

Note that property (a) in Lemma 3.5 guarantees that $c \in\left[0, \mu_{1}\left(X^{l}\right)\right]$. 
Lemma 3.7. Under Assumption 3.4, the sets $L_{a} \in \mathcal{F}, a \in\left[0, \mu_{1}(X)\right]$, have the following properties: (a) $\mu_{1}\left(L_{a}\right)=a$; (b) $\left\{f(x)<l_{a}\right\} \subseteq L_{a} \subseteq\left\{f(x) \leq l_{a}\right\}$; (c) $L_{a} \subset L_{a^{\prime}} \subseteq X$ if $a<a^{\prime} \leq \mu_{1}(X)$.

Proof. For (a), $\mu_{1}\left(L_{a}\right)=\mu_{1}\left(\left\{f(x)<l_{a}\right\}\right)+\mu_{1}\left(W_{c}\left(X^{l_{a}}\right)\right)=\mu_{1}\left(\left\{f(x)<l_{a}\right\}\right)+$ $a-\mu_{1}\left(\left\{f(x)<l_{a}\right\}\right)=a$. Property (b) follows from $W_{c}\left(X^{l_{a}}\right) \subseteq X^{l_{a}}$ and (3.4). For (c), if $l_{a}=l_{a^{\prime}}$, then $c<c^{\prime}$, where $c^{\prime}=a^{\prime}-\mu_{1}\left(\left\{f(x)<l_{a}\right\}\right)$, and thus

$$
L_{a}=\left\{f(x)<l_{a}\right\} \cup W_{c}\left(X^{l_{a}}\right) \subset\left\{f(x)<l_{a}\right\} \cup W_{c^{\prime}}\left(X^{l_{a}}\right)=L_{a^{\prime}} .
$$

If $l_{a}<l_{a^{\prime}}$, then $L_{a} \subseteq\left\{f(x) \leq l_{a}\right\} \subset\left\{f(x)<l_{a^{\prime}}\right\} \subseteq L_{a^{\prime}}$.

Let $M_{a, d}=L_{a+d} \backslash L_{a}$. For each $d \in\left[0, \mu_{1}(X)\right]$ and each $a \in\left[0, \mu_{1}(X)-d\right]$, denote $g_{d}(a)=\mu_{2}\left(M_{a, d}\right)=\int_{M_{a, d}} f(x) \mu_{1}(d x)$. The function $g_{d}(a)$ is nondecreasing and continuous in $a \in\left[0, \mu_{1}(X)-d\right]$ for each $d \in\left[0, \mu_{1}(X)\right]$. However, we will not use the fact that it is nondecreasing. So we only prove the continuity in the following lemma.

Lemma 3.8. Under Assumption 3.4, $g_{d}(a)$ is continuous in $a \in\left[0, \mu_{1}(X)-d\right]$ for each $d \in\left[0, \mu_{1}(X)\right]$.

Proof. We show that $\mu_{2}\left(L_{a+d}\right)$ is continuous in $a \in\left(\left[0, \mu_{1}(X)-d\right]\right.$ for any $d \in$ $\left[0, \mu_{1}(X)\right]$. Since $g_{d}(a)=L_{a+d}-L_{a}$, this implies the lemma. Consider a sequence $\left\{a^{k}: k=1,2, \ldots\right\}$, where $a_{k} \in\left[0, \mu_{1}(X)-d\right]$. Let $a^{k} \nearrow a$. Then $L_{a^{k}+d} \subset L_{a^{k+1}+d} \subset$ $B \subseteq L_{a+d}$, where $B=\bigcup_{k=1}^{\infty} L_{a^{k}+d}$. Therefore, $\mu_{i}\left(L_{a^{k}+d}\right) \nearrow \mu_{i}(B)$ and $\mu_{i}\left(L_{a+d}\right)=$ $\mu_{i}(B)+\mu_{i}\left(L_{a+d} \backslash B\right), i=1,2$. Since $\mu_{1}\left(L_{a^{k}+d}\right)=a^{k}+d \nearrow a+d=\mu_{1}\left(L_{a+d}\right)$, we have $\mu_{1}(B)=a+d$ and $\mu_{1}\left(L_{a+d} \backslash B\right)=0$. Since $\mu_{1}$ and $\mu_{2}$ are equivalent measures, $\mu_{2}\left(L_{a+d} \backslash B\right)=0$ and $\mu_{2}\left(L_{a^{k}+d}\right) \nearrow \mu_{2}\left(L_{a+d}\right)$.

Now let $a^{k} \searrow a$. Then $L_{a^{k}+d} \supset L_{a^{k+1}+d} \supset D \supseteq L_{a+d}$, where $D=\bigcap_{k=1}^{\infty} L_{a^{k}+d}$, and $\mu_{i}\left(L_{a^{k}+d}\right) \searrow \mu_{i}(D), \mu_{i}\left(L_{a+d}\right)=\mu_{i}(D)-\mu_{i}\left(D \backslash L_{a+d}\right)$ for $i=1$, 2. Similar to the previous case, $\mu_{1}\left(L_{a^{k}+d}\right)=a^{k}+d \searrow a+d=\mu_{1}\left(L_{a+d}\right)$, so $\mu_{1}(D)=a+d$, $\mu_{2}\left(D \backslash L_{a+d}\right)=\mu_{1}\left(D \backslash L_{a+d}\right)=0$, and $\mu_{2}(D)=\mu_{2}\left(L_{a+d}\right)$. Thus, $\mu_{2}\left(L_{a^{k}+d}\right) \searrow$ $\mu_{2}\left(L_{a+d}\right)$.

Observe that a point $q \in \mathbb{R}^{2}$ is on the upper (lower) boundary of $R_{\mu}(X)$ if and only if $q \in R_{\mu}(X)$ and $q_{2}^{\prime} \leq q_{2}\left(q_{2}^{\prime} \geq q_{2}\right)$ for any $q^{\prime} \in R_{\mu}(X)$ with $q_{1}^{\prime}=q_{1}$.

Lemma 3.9. Under Assumption 3.4, a point $q \in \mathbb{R}^{2}$ is on the lower boundary of $R_{\mu}(X)$ if and only if $0 \leq q_{1} \leq \mu_{1}(X)$ and $q_{2}=\mu_{2}\left(L_{q_{1}}\right)$, and it is on the upper boundary of $R_{\mu}(X)$ if and only if $0 \leq q_{1} \leq \mu_{1}(X)$ and $q_{2}=\mu_{2}\left(X \backslash L_{\mu_{1}(X)-q_{1}}\right)$.

Proof. For the lower boundary, let $q_{2}=\mu_{2}\left(L_{q_{1}}\right)$. Since $q_{1}=\mu_{1}\left(L_{q_{1}}\right)$, we have $q=\mu\left(L_{q}\right) \in R_{\mu}(X)$. For any set $Z \in \mathcal{F}$ with $\mu_{1}(Z)=q_{1}$, define disjoint sets $Z_{1}=Z \backslash L_{q_{1}}, Z_{2}=L_{q_{1}} \backslash Z$, and $M=Z \cap L_{q_{1}}$. Then $Z=Z_{1} \cup M, L_{q_{1}}=Z_{2} \cup M$, and $\mu_{1}\left(Z_{1}\right)=\mu_{1}\left(Z_{2}\right)$, since $\mu_{1}(Z)=q_{1}=\mu_{1}\left(L_{q_{1}}\right)$. Furthermore, $Z_{1} \subseteq\left\{f(x) \geq l_{q_{1}}\right\}$ and $Z_{2} \subseteq\left\{f(x) \leq l_{q_{1}}\right\}$. Therefore,

$$
\begin{aligned}
\mu_{2}\left(Z_{1}\right) & =\int_{Z_{1}} f(x) \mu_{1}(d x) \geq l_{q_{1}} \int_{Z_{1}} \mu_{1}(d x) \\
& =l_{q_{1}} \int_{Z_{2}} \mu_{1}(d x) \geq \int_{Z_{2}} f(x) \mu_{1}(d x)=\mu_{2}\left(Z_{2}\right) .
\end{aligned}
$$

So $\mu_{2}(Z)=\mu_{2}\left(Z_{1}\right)+\mu_{2}(M) \geq \mu_{2}\left(Z_{2}\right)+\mu_{2}(M)=\mu_{2}\left(L_{q_{1}}\right)$, and thus $q$ is on the lower boundary of $R_{\mu}(X)$. 
If $q$ is on the lower boundary of $R_{\mu}(X)$, then $q_{2} \leq \mu_{2}\left(L_{q_{1}}\right)$. Since $q \in R_{\mu}(X)$, there exists $Z \in \mathcal{F}$ with $\mu(Z)=q$. But, as proved above, $\mu_{2}(Z) \geq \mu_{2}\left(L_{q_{1}}\right)$ for any $Z \in \mathcal{F}$ with $\mu_{1}(Z)=q_{1}$. Thus $q_{2} \geq \mu_{2}\left(L_{q_{1}}\right)$. Therefore, $q_{2}=\mu_{2}\left(L_{q_{1}}\right)$.

The statement on the upper boundary follows from the symmetry of $R_{\mu}(X)$.

Lemma 3.10. Under Assumption 3.4, given $u=\left(u_{1}, u_{2}\right) \in R_{\mu}(X)$, there exists $a^{*} \in\left[0, \mu_{1}(X)-u_{1}\right]$ such that $\mu\left(M_{a^{*}, u_{1}}\right)=u$.

Proof. Since $\mu_{1}\left(L_{0}\right)=0$ and $\mu_{1}$ and $\mu_{2}$ are equivalent, $\mu_{2}\left(L_{0}\right)=0$. Therefore, $g_{u_{1}}(0)=\mu_{2}\left(L_{u_{1}} \backslash L_{0}\right)=\mu_{2}\left(L_{u_{1}}\right)-\mu_{2}\left(L_{0}\right)=\mu_{2}\left(L_{u_{1}}\right)$. Similarly, $\mu_{2}\left(X \backslash L_{\mu_{1}(X)}\right)$ $=0$, because $\mu_{1}\left(X \backslash L_{\mu_{1}(X)}\right)=\mu_{1}(X)-\mu_{1}\left(L_{\mu_{1}(X)}\right)=0$. Thus

$$
\begin{aligned}
g_{u_{1}}\left(\mu_{1}(X)-u_{1}\right) & =\mu_{2}\left(L_{\mu_{1}(X)} \backslash L_{\mu_{1}(X)-u_{1}}\right)=\mu_{2}\left(L_{\mu_{1}(X)}\right)-\mu_{2}\left(L_{\mu_{1}(X)-u_{1}}\right) \\
& =\mu_{2}(X)-\mu_{2}\left(X \backslash L_{\mu_{1}(X)}\right)-\mu_{2}\left(L_{\mu_{1}(X)-u_{1}}\right) \\
& =\mu_{2}\left(X \backslash L_{\mu_{1}(X)-u_{1}}\right) .
\end{aligned}
$$

According to Lemma 3.9. the point $\left(u_{1}, g_{u_{1}}(0)\right)$ is on the lower boundary of the range $R_{\mu}(X)$ and the point $\left(u_{1}, g_{u_{1}}\left(\mu_{1}(X)-u_{1}\right)\right)$ is on the upper boundary of the range $R_{\mu}(X)$. So $u_{2} \in\left[g_{u_{1}}(0), g_{u_{1}}\left(\mu_{1}(X)-u_{1}\right)\right]$. Since $g_{u_{1}}(a)$ is continuous in $a \in\left[0, \mu_{1}(X)-u_{1}\right]$, there exists $a^{*}$, such that $g_{u_{1}}\left(a^{*}\right)=u_{2}$. That is, $\mu_{2}\left(M_{a^{*}, u_{1}}\right)=$ $u_{2}$. By definition, $\mu_{1}\left(M_{a^{*}, u_{1}}\right)=u_{1}$. Therefore, $\mu\left(M_{a^{*}, u_{1}}\right)=u$.

Note that Lemmas 3.5 and $3.7 \sqrt[3.10]{ }$ hold if one replaces everywhere the set $X$ with any measurable subset $Z \in \mathcal{F}$. In particular, expressions such as $\{f(x)<l\}$ should be replaced with $\{x \in Z: f(x)<l\}$. We define explicitly

$$
l_{a}(Z)=\min \left\{l \geq 0: \mu_{1}(\{x \in Z: f(x) \leq l\}) \geq a\right\} .
$$

Let $Z^{l}=\{x \in Z: f(x)=l\}$. As follows from Ross [10, Theorem 2(LT3)], for each $l \in[0, \infty)$, there exists a family

$$
\left\{W_{b}\left(Z^{l}\right) \in \mathcal{F}_{Z^{l}}: b \in\left[0, \mu_{1}\left(Z^{l}\right)\right]\right\}
$$

such that $W_{b}\left(Z^{l}\right) \subset W_{b^{\prime}}\left(Z^{l}\right) \subseteq Z^{l}$ whenever $b<b^{\prime} \leq \mu_{1}\left(Z^{l}\right)$ and $\mu_{1}\left(W_{b}\left(Z^{l}\right)\right)=$ $b$ for each $b \in\left[0, \mu_{1}\left(Z^{l}\right)\right]$. Again, we fix a family of $W_{b}\left(Z^{l}\right)$ for each $l \in[0, \infty)$ and each $Z$, and define

$$
L_{a}(Z)=\left\{x \in Z: f(x)<l_{a}\right\} \cup W_{c}\left(Z^{l_{a}}\right),
$$

where $c=a-\mu_{1}(\{x \in Z: f(x)<a\})$. Note that $l_{a}(X)=l_{a}$ and $L_{a}(X)=L_{a}$, for each $a \in\left[0, \mu_{1}(X)\right]$. In the following two lemmas and their proofs, for a given $u \in R_{\mu}(X)$, we consider a point $a^{*} \in\left[0, \mu_{1}(X)-u_{1}\right]$ with $\mu\left(M_{a^{*}, u_{1}}\right)=u$ and the set $Z=X \backslash M_{a^{*}, u_{1}}$. The existence of $a^{*}$ is stated in Lemma 3.10. Later it will become clear that $Z$ is the maximal subset with the vector measure $p=\mu(X)-u$ and $M_{a^{*}, u_{1}}$ is the the minimal subset with the vector measure $p=u$.

Lemma 3.11. Let Assumption 3.4 hold. For a given $u=\left(u_{1}, u_{2}\right) \in R_{\mu}(X)$, consider $a^{*} \in\left[0, \mu_{1}(X)-u_{1}\right]$ with $\mu\left(M_{a^{*}, u_{1}}\right)=u$. Then

$$
\mu_{2}\left(L_{a}(Z)\right)= \begin{cases}\mu_{2}\left(L_{a}\right) & \text { if } a \in\left[0, a^{*}\right] ; \\ \mu_{2}\left(L_{a+u_{1}} \backslash M_{a^{*}, u_{1}}\right) & \text { if } a \in\left(a^{*}, \mu_{1}(X)-u_{1}\right] .\end{cases}
$$


Proof. First, consider the case $a \in\left[0, a^{*}\right]$. We have $Z=X \backslash M_{a^{*}, u_{1}} \supseteq L_{a^{*}} \supseteq L_{a}=$ $\left\{f(x)<l_{a}\right\} \cup W_{c}\left(X^{l_{a}}\right)$, where $c=a-\mu_{1}\left(\left\{f(x)<l_{a}\right\}\right)$. In addition, $\left\{f(x)<l_{a}\right\} \cup$ $W_{c}\left(X^{l_{a}}\right) \subseteq\left\{f(x) \leq l_{a}\right\}$. Therefore

$$
\begin{aligned}
& \mu_{1}\left(\left\{x \in Z: f(x) \leq l_{a}\right\}\right)=\mu_{1}\left(Z \cap\left\{f(x) \leq l_{a}\right\}\right) \\
& \quad \geq \mu_{1}\left(Z \cap\left(\left\{f(x)<l_{a}\right\} \cup W_{c}\left(X^{l_{a}}\right)\right)\right)=\mu_{1}\left(\left\{f(x)<l_{a}\right\} \cup W_{c}\left(X^{l_{a}}\right)\right)=a .
\end{aligned}
$$

Thus, (3.5) implies that $l_{a}(Z) \leq l_{a}$. On the other hand, take an arbitrary $l<l_{a}$. Since $Z \subseteq X$,

$$
\mu_{1}(\{x \in Z: f(x) \leq l\}) \leq \mu_{1}(\{f(x) \leq l\})<a .
$$

Therefore, $l_{a}(Z)>l$ for all $l<l_{a}$. Thus, $l_{a}(Z) \geq l_{a}$. We conclude that $l_{a}(Z)=l_{a}$.

Denote $A=\left\{f(x)<l_{a}\right\}$. Since $Z \supseteq L_{a} \supseteq A$ and $l_{a}(Z)=l_{a}$, then $\{x \in$ $\left.Z: f(x)<l_{a}(Z)\right\}=A$. By definition, each of the sets $L_{a}$ and $L_{a}(Z)$ is the union of two disjoint subsets: $L_{a}=A \cup W_{c}\left(X^{l_{a}}\right)$ and $L_{a}(Z)=A \cup W_{b}\left(Z^{l_{a}}\right)$ with $c=a-\mu_{1}(A)=b$. Thus, since $X^{l_{a}} \supseteq Z^{l_{a}}$ and $f(x)=l_{a}$ when $x \in X^{l_{a}}$, we have $\mu_{2}\left(W_{c}\left(X^{l_{a}}\right)\right)=\mu_{2}\left(W_{c}\left(Z^{l_{a}}\right)\right)=l_{a} c$. So, $\mu_{2}\left(L_{a}(Z)\right)=\mu_{2}(A)+\mu_{2}\left(W_{c}\left(Z^{l_{a}}\right)\right)=$ $\mu_{2}(A)+\mu_{2}\left(W_{c}\left(X^{l_{a}}\right)\right)=\mu_{2}\left(L_{a}\right)$.

Second, consider the case $a \in\left(a^{*}, \mu_{1}(X)-u_{1}\right]$. Observe that $M_{a^{*}, u_{1}} \subseteq L_{a^{*}+u_{1}} \subset$ $L_{a+u_{1}}=\left\{f(x)<l_{a+u_{1}}\right\} \cup W_{c}\left(X^{l_{a+u_{1}}}\right)$, where $c=a+u_{1}-\mu_{1}\left(\left\{f(x)<l_{a+u_{1}}\right\}\right)$. In addition, $\left\{f(x)<l_{a+u_{1}}\right\} \cup W_{c}\left(X^{l_{a+u_{1}}}\right) \subseteq\left\{f(x) \leq l_{a+u_{1}}\right\}$. Therefore,

$$
\begin{aligned}
& \mu_{1}\left(\left\{x \in Z: f(x) \leq l_{a+u_{1}}\right\}\right) \\
& \quad=\mu_{1}\left(\left\{f(x) \leq l_{a+u_{1}}\right\} \cap Z\right)=\mu_{1}\left(\left\{f(x) \leq l_{a+u_{1}}\right\} \backslash M_{a^{*}, u_{1}}\right) \\
& \quad \geq \mu_{1}\left(\left\{f(x)<l_{a+u_{1}}\right\} \cup W_{c}\left(X^{l_{a+u_{1}}}\right) \backslash M_{a^{*}, u_{1}}\right)=a+u_{1}-u_{1}=a .
\end{aligned}
$$

Thus, (3.5) implies that $l_{a}(Z) \leq l_{a+u_{1}}$. On the other hand, note that $M_{a^{*}, u_{1}} \subseteq$ $\left\{f(x) \leq l_{a}(Z)\right\}$. Indeed, since $a>a^{*}$, we have $l_{a}(Z) \geq l_{a^{*}}(Z)=l_{a^{*}}$. Assume $l_{a^{*}} \leq$ $l_{a}(Z)<l_{a^{*}+u_{1}}$. Then $\left\{x \in Z: f(x) \leq l_{a}(Z)\right\}=\left\{f(x) \leq l_{a}(Z)\right\} \backslash M_{a^{*}, u_{1}}=L_{a^{*}}$, and $a=\mu_{1}\left(\left\{x \in Z: f(x) \leq l_{a}(Z)\right\}\right)=\mu_{1}\left(L_{a^{*}}\right)=a^{*}$, which is a contradiction. Therefore, $l_{a}(Z) \geq l_{a^{*}+u_{1}}$ and $M_{a^{*}, u_{1}} \subseteq\left\{f(x) \leq l_{a^{*}+u_{1}}\right\} \subseteq\left\{f(x) \leq l_{a}(Z)\right\}$. Thus, $\left\{x \notin Z: f(x) \leq l_{a}(Z)\right\}=\left\{x \in M_{a^{*}, u_{1}}: f(x) \leq l_{a}(Z)\right\}=M_{a^{*}, u_{1}}$ and

$$
\mu_{1}\left(\left\{f(x) \leq l_{a}(Z)\right\}\right)=\mu_{1}\left(\left\{x \in Z: f(x) \leq l_{a}(Z)\right\}\right)+\mu_{1}\left(M_{a^{*}, u_{1}}\right) \geq a+u_{1},
$$

where the last step follows from property (b) in Lemma 3.7. Formula (3.1) implies that $l_{a}(Z) \geq l_{a+u_{1}}$. Therefore, $l_{a}(Z)=l_{a+u_{1}}$.

Consider again the identity $L_{a+u_{1}}=\left\{f(x)<l_{a+u_{1}}\right\} \cup W_{c}\left(X^{l_{a}+u_{1}}\right)$, where the sets in the union are disjoint and $c=\left(a+u_{1}\right)-\mu_{1}\left(\left\{f(x)<l_{a+u_{1}}\right\}\right)$. Similarly, $L_{a}(Z)=\left\{x \in Z: f(x)<l_{a+u_{1}}\right\} \cup W_{b}\left(Z^{l_{a}+u_{1}}\right)$, where $b=a-\mu_{1}(\{x \in Z:$ $\left.\left.f(x)<l_{a}(Z)\right\}\right)$. Since $l_{a}(Z)=l_{a+u_{1}}$ and $\left\{f(x)<l_{a+u_{1}}\right\} \supset M_{a^{*}, u_{1}}$, we have $b=a-\mu_{1}\left(\left\{x \in Z: f(x)<l_{a+u_{1}}\right\}\right)=a-\mu_{1}\left(\left\{f(x)<l_{a+u_{1}}\right\} \backslash M_{a^{*}, u_{1}}\right)=\left(a+u_{1}\right)-$ $\mu_{1}\left(\left\{f(x)<l_{a+u_{1}}\right\}\right)=c$. Thus,

$$
\begin{aligned}
\mu_{2}\left(L_{a}(Z)\right) & =\mu_{2}\left(\left\{x \in Z: f(x)<l_{a}(Z)\right\}\right)+\mu_{2}\left(W_{b}\left(Z^{l_{a}(Z)}\right)\right) \\
& =\mu_{2}\left(\left\{x \in Z: f(x)<l_{a+u_{1}}\right\}\right)+l_{a+u_{1}} \mu_{1}\left(W_{b}\left(Z^{l_{a+u_{1}}}\right)\right) \\
& =\mu_{2}\left(\left\{f(x)<l_{a+u_{1}}\right\}\right)-\mu_{2}\left(M_{a^{*}, u_{1}}\right)+l_{a+u_{1}} \mu_{1}\left(W_{c}\left(X^{l_{a+u_{1}}}\right)\right) \\
& =\mu_{2}\left(L_{a+u_{1}}\right)-\mu_{2}\left(M_{a^{*}, u_{1}}\right)=\mu_{2}\left(L_{a+u_{1}} \backslash M_{a^{*}, u_{1}}\right),
\end{aligned}
$$

where the second equality holds because $l_{a}(Z)=l_{a+u_{1}}, f(x)=l_{a+u_{1}}$ for $x \in X^{l_{a+u_{1}}}$, and $Z^{l_{a+u_{1}}} \subseteq X^{l_{a+u_{1}}}$ (in fact $Z^{l_{a+u_{1}}}=X^{l_{a+u_{1}}}$, but we do not use this). The 
third equality holds because of $\left\{x \in Z: f(x)<l_{a+u_{1}}\right\}=\left\{f(x)<l_{a+u_{1}}\right\} \backslash$ $M_{a^{*}, u_{1}},\left\{f(x)<l_{a+u_{1}}\right\} \supset M_{a^{*}, u_{1}}$, and $b=c$. The fourth equality follows from $l_{a+u_{1}} \mu_{1}\left(W_{c}\left(X^{l_{a+u_{1}}}\right)\right)=\mu_{2}\left(W_{c}\left(X^{l_{a+u_{1}}}\right)\right)$.

Lemma 3.12. Let Assumption 3.4 hold. For a given $u=\left(u_{1}, u_{2}\right) \in R_{\mu}(X)$, consider $a^{*} \in\left[0, \mu_{1}(X)-u_{1}\right]$ with $\mu\left(M_{a^{*}, u_{1}}\right)=u$. Let $q=\left(q_{1}, q_{2}\right)$ be on the lower (upper) boundary of $R_{\mu}(Z)$. If $q_{1} \in\left[0, a^{*}\right]\left(q_{1} \in\left[0, \mu_{1}(X)-u_{1}-a^{*}\right)\right)$, then $q$ is on the lower (upper) boundary of $R_{\mu}(X)$, and if $q_{1} \in\left(a^{*}, \mu_{1}(X)-u_{1}\right]$ $\left(q_{1} \in\left[\mu_{1}(X)-u_{1}-a^{*}, \mu_{1}(X)-u_{1}\right]\right)$, then $r=\mu(X)-u-q$ is on the upper (lower) boundary of $R_{\mu}(X)$.

Proof. When $q$ is on the lower boundary of $R_{\mu}(Z)$, according to Lemma 3.9, $\mu_{2}\left(L_{q_{1}}(Z)\right)=q_{2}$. If $q_{1} \in\left[0, a^{*}\right]$, then by Lemma 3.11, $\mu_{2}\left(L_{q_{1}}\right)=\mu_{2}\left(L_{q_{1}}(Z)\right)=q_{2}$, and Lemma 3.9 implies that $q$ is on the lower boundary of $R_{\mu}(X)$.

If $q_{1} \in\left(a^{*}, \mu(X)-u_{1}\right]$, then for $r=\left(r_{1}, r_{2}\right)$,

$$
\begin{aligned}
r_{2} & =\mu_{2}(X)-u_{2}-q_{2}=\mu_{2}(X)-\mu_{2}\left(M_{a^{*}, u_{1}}\right)-\mu_{2}\left(L_{q_{1}}(Z)\right) \\
& =\mu_{2}(X)-\left(\mu_{2}\left(M_{a^{*}, u_{1}}\right)+\mu_{2}\left(L_{q_{1}+u_{1}} \backslash M_{a^{*}, u_{1}}\right)\right)=\mu_{2}(X)-\mu_{2}\left(L_{q_{1}+u_{1}}\right) \\
& =\mu_{2}\left(X \backslash L_{q_{1}+u_{1}}\right)=\mu_{2}\left(X \backslash L_{\mu_{1}(X)-r_{1}}\right),
\end{aligned}
$$

where the first and last equalities follow from the definition of $r$, the second equality follows from Lemma 3.10, the third equality follows from Lemma 3.11, and the fourth equality follows from $q_{1}>a^{*}$. According to Lemma 3.9 $r$ is on the upper boundary of $R_{\mu}(X)$.

If $q$ is on the upper boundary of $R_{\mu}(Z)$, then, because of symmetry, $r=\mu(X)-$ $u-q$ is on the lower boundary of $R_{\mu}(Z)$. If $q_{1} \in\left[\mu_{1}(X)-u_{1}-a^{*}, \mu_{1}(X)-u_{1}\right]$, then $\mu_{1}(X)-u_{1}-q_{1} \in\left[0, a^{*}\right]$. From the first part of the proof, $r=\mu(X)-u-q$ is on the lower boundary of $R_{\mu}(X)$. If $q_{1} \in\left[0, \mu_{1}(X)-u_{1}-a^{*}\right)$, then $\mu_{1}(X)-u_{1}-q_{1} \in$ $\left(a^{*}, \mu_{1}(X)-u_{1}\right]$. Again, from the first part of the proof, $\mu(X)-u-\left(\mu(X)-u-q_{1}\right)=$ $q_{1}$ is on the upper boundary of $R_{\mu}(X)$.

Lemma 3.13. Under Assumption 3.4, for any vector $p \in R_{\mu}(X)$, there exists a maximal set $Z^{*} \in \mathcal{S}_{p}(X)$ and, in addition, $R_{\mu}^{p}(X)=Q_{\mu}^{p}(X)$.

Proof. For $u=\mu(X)-p$, consider $a^{*}$ defined in Lemma 3.10 For $Z^{*}=X \backslash$ $M_{a^{*}, \mu_{1}(X)-p_{1}}$, the following three statements are true: (1) $R_{\mu}\left(Z^{*}\right) \subseteq R_{\mu}^{p}(X) ;(2)$ $R_{\mu}^{p}(X) \subseteq Q_{\mu}^{p}(X) ;(3) Q_{\mu}^{p}(X) \subseteq R_{\mu}\left(Z^{*}\right)$.

For (1), $\mu\left(Z^{*}\right)=\mu\left(X \backslash M_{a^{*}, \mu_{1}(X)-p_{1}}\right)=\mu(X)-\mu\left(M_{a^{*}, \mu_{1}(X)-p_{1}}\right)=\mu(X)-$ $(\mu(X)-p)=p$, where the second to the last equality follows from Lemma 3.10 . Thus, $R_{\mu}\left(Z^{*}\right)=R_{\mu}^{p}(X)$.

For (2), assume that there exists a vector $q \in R_{\mu}^{p}(X)$ such that $q \notin Q_{\mu}^{p}(X)$. Then Definition 2.1 implies that either $q \notin R_{\mu}(X)$ or $q \notin\left(R_{\mu}(X)-\{\mu(X)-p\}\right)$. However $q \in R_{\mu}^{p}(X) \subseteq R_{\mu}(X)$. Therefore, $q \notin R_{\mu}(X)-\{\mu(X)-p\}$, which is equivalent to $p-q \notin\{\mu(X)\}-R_{\mu}(X)=R_{\mu}(X)$, where the equality follows from Lemma 3.1. Since $R_{\mu}^{p}(X) \subseteq R_{\mu}(X)$, we have $p-q \notin R_{\mu}^{p}(X)$. By Lemma 3.3. $R_{\mu}^{p}(X)$ is centrally symmetric with the center $\frac{p}{2}$. Therefore $q \notin R_{\mu}^{p}(X)$. The above contradiction implies (2).

For (3), assume $q \in Q_{\mu}^{p}(X)$, but $q \notin R_{\mu}\left(Z^{*}\right)$. By Lyapunov's theorem, $R_{\mu}\left(Z^{*}\right)$ is a convex compactum. Let $q^{u}=\left(q_{1}, q_{2}^{u}\right)$ and $q^{l}=\left(q_{1}, q_{2}^{l}\right)$ be the intersection points of the vertical line $\mu_{1}=q_{1}$ and the upper and lower boundaries of $R_{\mu}\left(Z^{*}\right)$ respectively. Then one of the following must be true: $q_{2}>q_{2}^{u}$ or $q_{2}<q_{2}^{l}$. Without 
loss of generosity, we consider the former case. Since $q_{u}$ is on the upper boundary of $R_{\mu}\left(Z^{*}\right)$, according to Lemma 3.12, one of the following is true: (a) $q^{u}$ is on the upper boundary of $R_{\mu}(X)$ or (b) $r=p-q^{u}$ is on the lower boundary of $R_{\mu}(X)$. For (a), $q_{2}>q_{2}^{u}$ implies $q \notin R_{\mu}(X)$. Thus $q \notin Q_{\mu}^{p}(X)$. This contradicts our assumption. For (b), we let $r^{\prime}=p-q$. Obviously, $r_{1}^{\prime}=r_{1}$ and $r_{2}^{\prime}<r_{2}$. This implies that $r^{\prime}$ is below the lower boundary point $r$. Thus, $r^{\prime} \notin R_{\mu}(X)$ and $r^{\prime} \notin Q_{\mu}^{p}(X)$. But according to Lemma 3.3. this means $q \notin Q_{\mu}^{p}(X)$, which contradicts our assumption. Statements (1)-(3) imply the lemma.

Let $D$ be a two-by-two invertible matrix with positive entries, and $A \subseteq \mathbb{R}^{2}$. We denote by $A D$ the set $\{p D: p \in A\}$. For a vector measure $\mu=\left(\mu_{1}, \mu_{2}\right)$, let $\nu=\mu D$ be the vector measure $\left(\nu_{1}, \nu_{2}\right)=\left(D_{11} \mu_{1}+D_{21} \mu_{2}, D_{12} \mu_{1}+D_{22} \mu_{2}\right)$. Then the measures $\nu_{1}$ and $\nu_{2}$ are equivalent.

Lemma 3.14. (a) $R_{\mu}(Y) D=R_{\nu}(Y)$ for all $Y \in \mathcal{F}$; (b) $R_{\mu}^{p}(X) D=R_{\nu}^{p D}(X)$ for all $p \in R_{\mu}(X)$; (c) $Q_{\mu}^{p}(X) D=Q_{\nu}^{p D}(X)$ for all $p \in R_{\mu}(X)$.

Proof. (a) For any point $q \in R_{\nu}(Y)$, there exists a set $Z \in \mathcal{F}_{Y}$ such that $\nu(Z)=q$. Since $\mu(Z)=q D^{-1}$ and $q D^{-1} \in R_{\mu}(Y)$, we have $q \in R_{\mu}(Y) D$. For any point $q \in R_{\mu}(Y) D$, we have $q D^{-1} \in R_{\mu}(Y)$. Thus there exists a set $Z \in \mathcal{F}_{Y}$ such that $\mu(Z)=q D^{-1}$ and $\nu(Z)=q$. Therefore, $\nu(Z) \in R_{\nu}(Y)$.

(b) For any point $q \in R_{\nu}^{p D}(X)$, there exist sets $Y \in \mathcal{F}$ and $Z \in \mathcal{F}_{Y}$ such that $\nu(Y)=p D$ and $\nu(Z)=q$. So $\mu(Y)=p$ and $\mu(Z)=q D^{-1}$. Thus, $q D^{-1} \in R_{\mu}^{p}(X)$ and therefore, $q \in R_{\mu}^{p}(X) D$. For any point $q \in R_{\mu}^{p}(X) D$, we have $q D^{-1} \in R_{\mu}^{p}(X)$. So there exist sets $Y \in \mathcal{F}$ and $Z \in \mathcal{F}_{Y}$ such that $\mu(Y)=p$ and $\mu(Z)=q D^{-1}$, and consequently $\nu(Y)=p D$ and $\nu(Z)=q$. Thus $q \in R_{\mu}^{p D}(X)$.

(c) According to Definition 2.1. $Q_{\mu}^{p}(X) D=\left(R_{\mu}(X) D-\{\mu(X) D-p D\}\right) \cap$ $R_{\mu}(X) D=\left(R_{\nu}(X)-\{\nu(X)-p D\}\right) \cap R_{\nu}(X)=Q_{\nu}^{p D}(X)$.

Proof of Theorem 2.3. According to Lemma 3.13, Theorem 2.3 holds under Assumption 3.4. which states that $\mu_{1}$ and $\mu_{2}$ are equivalent. If $\mu_{1}$ and $\mu_{2}$ are not equivalent, consider $\nu=\mu D$. Since $\nu_{1}$ and $\nu_{2}$ are equivalent, $Q_{\mu}^{p}(X)=$ $Q_{\nu}^{p D}(X) D^{-1}=R_{\nu}^{p D}(X) D^{-1}=R_{\mu}^{p}(X)$, where the first equality and the last equality are by Lemma 3.14 and the second equality is due to Lemma 3.13. Furthermore, according to Lemma 3.13, there exists a maximal set $Z^{*}$ such that $R_{\nu}\left(Z^{*}\right)=R_{\nu}^{p D}(X)$. Therefore, $R_{\mu}\left(Z^{*}\right)=R_{\nu}\left(Z^{*}\right) D^{-1}=R_{\nu}^{p D}(X) D^{-1}=R_{\mu}^{p}(X)$.

Now consider Theorem 2.4. For $A \subseteq \mathbb{R}^{m}$ and $b \in \mathbb{R}^{m}$, let $A+b=\{a+b: a \in A\}$ and $A-b=A+(-b)$. Observe that $A-b=A-\{b\}$. Recall that $A \oplus B=$ $\bigcup_{b \in B}(A+b)$ is called the Minkowski addition and $A \ominus B=\bigcap_{b \in B}(A-b)$ is called the Minkowski subtraction, where $A, B \subseteq \mathbb{R}^{m}$.

Lemma 3.15. Let $A_{1}, A_{2}, B_{1}, B_{2} \subseteq \mathbb{R}^{2}$ be convex and compact sets such that $A_{1} \oplus$ $B_{1}=A_{2} \oplus B_{2}$ and $B_{1} \subseteq B_{2}$. Then $A_{2} \subseteq A_{1}$.

Proof. According to [11, Lemma 3.1.8], if $A, B \subseteq \mathbb{R}^{2}$ are convex and compact sets, then $(A \oplus B) \ominus B=A$. Thus if $a \in A_{2}$, then $a \in\left(A_{2} \oplus B_{2}\right) \ominus B_{2}$, and consequently $a \in\left(A_{1} \oplus B_{1}\right) \ominus B_{2}$. So $a \in\left(A_{1} \oplus B_{1}\right)-b$, for any $b \in B_{2}$. Since $B_{1} \subseteq B_{2}$, we have $a \in\left(A_{1} \oplus B_{1}\right)-b$, for any $b \in B_{1}$, and thus $a \in\left(A_{1} \oplus B_{1}\right) \ominus B_{1}=A_{1}$.

Proof of Theorem 2.4. Now let $Z^{*}$ be the maximal set with the measure $p$. Then $\mu\left(X \backslash Z^{*}\right)=\mu(X)-p$. Consider any set $M$ such that $\mu(M)=\mu(X)-p$. Obviously, 
$R_{\mu}(M) \oplus R_{\mu}(X \backslash M)=R_{\mu}\left(X \backslash Z^{*}\right) \oplus R_{\mu}\left(Z^{*}\right)=R_{\mu}(X)$. In addition, $R_{\mu}(X \backslash M) \subseteq$ $R_{\mu}\left(Z^{*}\right)$ by definition. Thus according to Lemma 3.15, $R_{\mu}\left(X \backslash Z^{*}\right) \subseteq R_{\mu}(M)$.

Similarly, let $M^{*}=X \backslash Z^{*}$ be the minimal set with the measure $\mu(X)-p$. Then $\mu\left(Z^{*}\right)=p$. Consider any set $Z$ such that $\mu(Z)=p$. Obviously, $R_{\mu}(Z) \oplus$ $R_{\mu}(X \backslash Z)=R_{\mu}\left(Z^{*}\right) \oplus R_{\mu}\left(X \backslash Z^{*}\right)=R_{\mu}(X)$. In addition, $R_{\mu}\left(X \backslash Z^{*}\right)=$ $R_{\mu}\left(M^{*}\right) \subseteq R_{\mu}(X \backslash Z)$ by definition. Thus according to Lemma 3.15, $R_{\mu}(Z) \subseteq$ $R_{\mu}\left(Z^{*}\right)$.

With Theorem 2.4 the existence of the minimal subset $M^{*} \in \mathcal{S}_{\mu}^{q}(X)$ immediately follows from the existence of the maximal subset $Z^{*} \in \mathcal{S}_{\mu}^{\mu(X)-q}(X)$. Furthermore, $R_{\mu}\left(M^{*}\right)=\left(R_{\mu}\left(M^{*}\right) \oplus R_{\mu}\left(Z^{*}\right)\right) \ominus R_{\mu}\left(Z^{*}\right)=R_{\mu}(X) \ominus Q_{\mu}^{\mu(X)-q}(X)$.

Corollary 3.16. For a two-dimensional finite atomless vector measure $\mu=\left(\mu_{1}, \mu_{2}\right)$ and for a vector $q \in R_{\mu}(X)$, there exists a minimal set $M^{*} \in \mathcal{S}_{\mu}^{q}(X)$. In addition, $R_{\mu}\left(M^{*}\right)=R_{\mu}(X) \ominus Q_{\mu}^{\mu(X)-q}(X)$.

\section{Counterexample for 3D measures}

In this section, we present an example of a measurable space $(X, \mathcal{F})$ endowed with a three-dimensional atomless finite measure $\nu=\left(\nu_{1}, \nu_{2}, \nu_{3}\right)$ and a vector $p \in R_{\nu}(X)$ such that a maximal subset of $X$ with the measure $p$ does not exist. Theorem 2.4 implies that the minimum set does not exist either in this example.

Recall that, with respect to a measure $\mu$, sets $A$ and $B$ are said to be equal up to null sets (denoted by $A \simeq B$ ) if $\mu(A \backslash B)=\mu(B \backslash A)=0$. Also recall that $X^{l}=\{f(x)=l\}$.

Proposition 4.1. Let $\mu=\left(\mu_{1}, \mu_{2}\right)$ satisfy Assumption 3.4 and let $Y \in \mathcal{F}$. If $\mu_{1}\left\{X^{l_{\mu_{1}(Y)}}\right\}=0$ and $\mu_{2}(Y)=\mu_{2}\left(L_{\mu_{1}(Y)}\right)$, then $Y \simeq L_{\mu_{1}(Y)}$.

Proof. Assume that $Y \simeq L_{\mu_{1}(Y)}$ does not hold. We define three disjoint sets $Z_{1}=$ $Y \backslash L_{\mu_{1}(Y)}, Z_{2}=L_{\mu_{1}(Y)} \backslash Y$, and $M=Y \cap L_{\mu_{1}(Y)}$. Observe that $Y=Z_{1} \cup M$ and $L_{\mu_{1}(Y)}=Z_{2} \cup M$. These equalities and $\mu_{1}(Y)=\mu_{1}\left(L_{\mu_{1}(Y)}\right)$ imply $\mu_{1}\left(Z_{1}\right)=\mu_{1}\left(Z_{2}\right)$. Furthermore, $Z_{1} \subseteq\left\{f(x) \geq l_{\mu_{1}(Y)}\right\}$ and $Z_{2} \subseteq\left\{f(x)<l_{\mu_{1}(Y)}\right\}$, because according to (3.4), $L_{\mu_{1}(Y)}=\left\{f(x)<l_{\mu_{1}(Y)}\right\}$ when $\mu_{1}\left\{X^{l_{\mu_{1}(Y)}}\right\}=0$. Therefore,

$$
\begin{aligned}
\mu_{2}\left(Z_{1}\right) & =\int_{Z_{1}} f(x) \mu_{1}(d x) \geq l_{\mu_{1}(Y)} \int_{Z_{1}} \mu_{1}(d x) \\
& =l_{\mu_{1}(Y)} \int_{Z_{2}} \mu_{1}(d x)>\int_{Z_{2}} f(x) \mu_{1}(d x)=\mu_{2}\left(Z_{2}\right) .
\end{aligned}
$$

So $\mu_{2}(Y)=\mu_{2}\left(Z_{1}\right)+\mu_{2}(M)>\mu_{2}\left(Z_{2}\right)+\mu_{2}(M)=\mu_{2}\left(L_{\mu_{1}(Y)}\right)$. This contradiction implies the proposition.

Example 4.2. Let $X=[0,1]$ and $\mathcal{F}$ be the Borel $\sigma$-field. Consider the threedimensional vector measure $\nu(d x)=\left(\nu_{1}, \nu_{2}, \nu_{3}\right)(d x)=(1,2 x, \rho(x)) d x$, where

$$
\rho(x)= \begin{cases}4 x & \text { if } x \in\left[0, \frac{1}{2}\right) \\ 4 x-2 & \text { if } x \in\left[\frac{1}{2}, 1\right]\end{cases}
$$

Consider the points $p=\left(\frac{1}{2}, \frac{1}{2}, \frac{1}{2}\right), q^{1}=\left(\frac{1}{4}, \frac{1}{16}, \frac{1}{8}\right)$, and $q^{2}=\left(\frac{1}{4}, \frac{5}{32}, \frac{1}{16}\right)$. It is easy to show that $q^{1}, q^{2} \in R_{\nu}^{p}(X)$. Indeed let $Z^{1}=\left[0, \frac{1}{4}\right) \cup\left[\frac{3}{4}, 1\right], Z^{2}=\left[0, \frac{1}{8}\right) \cup$ $\left[\frac{3}{8}, \frac{5}{8}\right) \cup\left[\frac{7}{8}, 1\right], W^{1}=\left[0, \frac{1}{4}\right) \subseteq Z^{1}$, and $W^{2}=\left[0, \frac{1}{8}\right) \cup\left[\frac{1}{2}, \frac{5}{8}\right) \subseteq Z^{2}$, and we have $\nu\left(Z^{1}\right)=\nu\left(Z^{2}\right)=p, \nu\left(W^{1}\right)=q^{1}$, and $\nu\left(W^{2}\right)=q^{2}$. Since $Z^{1}$ and $Z^{2}$ are not 
equal up to a null set, Proposition 4.3 implies that there does not exist a set $Z$ such that $\nu(Z)=p$ and $q^{1}, q^{2} \in R_{\mu}(Z)$.

Proposition 4.3. Consider the sets $X, Z^{1}, Z^{2}$, the measure $\nu$ and vectors $p, q^{1}$, $q^{2}$ from Example 4.2, Let $Z \in \mathcal{S}_{\nu}^{p}(X)$. For each $i=1,2$, if $q^{i} \in R_{\nu}(Z)$, then $Z \simeq Z^{i}$.

Proof. Let $i=1$. Since $q^{1} \in R_{\nu}(Z)$, there exists a set $W^{1} \in \mathcal{F}_{Z}$ such that $\nu\left(W^{1}\right)=q^{1}$. Define a two-dimensional vector measure $\mu=\left(\mu_{1}, \mu_{2}\right)=\left(\nu_{1}, \nu_{2}\right)$. Then $\mu\left(W^{1}\right)=\left(\frac{1}{4}, \frac{1}{16}\right)$. Observe that, according to (3.1) and (3.4),$l_{\mu_{1}\left(W^{1}\right)}=l_{\frac{1}{4}}=$ $\frac{1}{2}$ and $L_{\mu_{1}\left(W^{1}\right)}=L_{\frac{1}{4}}=\left[0, \frac{1}{4}\right)$. In addition, $\mu_{1}\left(X^{l_{\mu_{1}\left(W^{1}\right)}}\right)=0$ and $\mu_{2}\left(W^{1}\right)=\frac{1}{16}=$ $\mu_{2}\left(L_{\mu_{1}\left(W^{1}\right)}\right)$. Therefore, according to Proposition 4.1, $W^{1} \simeq L_{\mu_{1}\left(W^{1}\right)}=\left[0, \frac{1}{4}\right)$. On the other hand, let $Y=W^{1} \cup(X \backslash Z)$. Since $W^{1} \subseteq Z, \nu(Y)=\nu\left(W^{1}\right)+$ $(\nu(X)-\nu(Z))=q^{1}+(\nu(X)-p)=\left(\frac{3}{4}, \frac{9}{16}, \frac{5}{8}\right)$, and thus, $\mu(Y)=\left(\frac{3}{4}, \frac{9}{16}\right)$. Observe that, according to (3.1) and (3.4), $l_{\mu_{1}(Y)}=l_{\frac{3}{4}}=\frac{3}{2}$ and $L_{\mu_{1}(Y)}=L_{\frac{3}{4}}=\left[0, \frac{3}{4}\right)$. In addition, $\mu_{1}\left(X^{\left.l_{\mu_{1}(Y)}\right)}=0\right.$ and $\mu_{2}(Y)=\frac{9}{16}=\mu_{2}\left(L_{\mu_{1}(Y)}\right)$. Therefore, according to Proposition 4.1 $Y \simeq L_{\mu_{1}(Y)}=L_{\frac{3}{4}}$. The above observations imply that $Z=W^{1} \cup(X \backslash Y) \simeq L_{\frac{1}{4}} \cup\left(X \backslash L_{\frac{3}{4}}\right)=\left[0, \frac{1}{4}\right) \cup\left([0,1] \backslash\left[0, \frac{3}{4}\right)\right)=\left[0, \frac{1}{4}\right) \cup\left[\frac{3}{4}, 1\right]=Z^{1}$.

Let $i=2$. Since $q^{2} \in R_{\nu}(Z)$, there exists a set $W^{2} \in \mathcal{F}_{Z}$ such that $\nu\left(W^{2}\right)=$ $q^{2}$. Define the two-dimensional vector measure $\mu=\left(\mu_{1}, \mu_{2}\right)=\left(\nu_{1}, \nu_{3}\right)$. Then $\mu\left(W^{2}\right)=\left(\frac{1}{4}, \frac{1}{16}\right)$. Observe that, according to (3.1) and (3.4), $l_{\mu_{1}\left(W^{2}\right)}=l_{\frac{1}{4}}=$ $\frac{1}{2}$ and $L_{\mu_{1}\left(W^{2}\right)}=L_{\frac{1}{4}}=\left[0, \frac{1}{8}\right) \cup\left[\frac{1}{2}, \frac{5}{8}\right)$. In addition, $\mu_{1}\left(X^{\left.l_{\mu_{1}\left(W^{2}\right)}\right)}=0\right.$ and $\mu_{2}\left(W^{2}\right)=\frac{1}{16}=\mu_{2}\left(L_{\mu_{1}\left(W^{2}\right)}\right)$. Therefore, according to Proposition 4.1, $W^{2} \simeq$ $L_{\mu_{1}\left(W^{2}\right)}=\left[0, \frac{1}{8}\right) \cup\left[\frac{1}{2}, \frac{5}{8}\right)$. On the other hand, let $Y=W^{2} \cup(X \backslash Z)$. Since $W^{2} \subseteq Z, \nu(Y)=\nu\left(W^{2}\right)+(\nu(X)-\nu(Z))=q^{2}+(\nu(X)-p)=\left(\frac{3}{4}, \frac{21}{32}, \frac{9}{16}\right)$, and thus, $\mu(Y)=\left(\frac{3}{4}, \frac{9}{16}\right)$. Observe that, according to (3.1) and (3.4), $l_{\mu_{1}(Y)}=l_{\frac{3}{4}}=\frac{3}{2}$ and $L_{\mu_{1}(Y)}=L_{\frac{3}{4}}=\left[0, \frac{3}{8}\right) \cup\left[\frac{1}{2}, \frac{7}{8}\right)$. In addition, $\mu_{1}\left(X^{\left.l_{\mu_{1}(Y)}\right)}=0\right.$ and $\mu_{2}(Y)=$ $\frac{9}{16}=\mu_{2}\left(L_{\mu_{1}(Y)}\right)$. Therefore, according to Proposition 4.1. $Y \simeq L_{\mu_{1}(Y)}=L_{\frac{3}{4}}$. The above observations imply that $Z=W^{2} \cup(X \backslash Y) \simeq L_{\frac{1}{4}} \cup\left(X \backslash L_{\frac{3}{4}}\right)=$ $\left(\left[0, \frac{1}{8}\right) \cup\left[\frac{1}{2}, \frac{5}{8}\right)\right) \cup\left([0,1] \backslash\left(\left[0, \frac{3}{8}\right) \cup\left[\frac{1}{2}, \frac{7}{8}\right)\right)\right)=\left[0, \frac{1}{8}\right) \cup\left[\frac{3}{8}, \frac{5}{8}\right) \cup\left[\frac{7}{8}, 1\right]=Z^{2}$.

\section{Geometric construction of maximal Ranges}

In 8], Lyapunov commented that a subset of the two-dimensional Euclidean space $\mathbb{R}^{2}$ is the range of some two-dimensional finite atomless vector measure on some measurable space if and only if it satisfies the following conditions: (1) it is convex; (2) it is closed; (3) it is centrally symmetric; (4) it contains the origin. Since the geometrically constructed set $Q_{\mu}^{p}(X)$ satisfies conditions (1)-(4), it must be the range of some two-dimensional finite atomless vector measure on some measurable space. Theorem 2.3 immediately tells us that it is the range of the vector measure $\mu$ on the measurable space $\left(Z^{*}, \mathcal{F}_{Z^{*}}\right)$. The second equality in Theorem 2.3 allows us to construct geometrically the set $R_{\mu}^{p}(x)$ by shifting the set $R_{\mu}(X)$ by $(p-\mu(X))$ and intersecting the shifted set with $R_{\mu}(X)$.

We consider three examples with the same set $X=[0,1]$, but with different probability vector measures. Let $p=(0.7,0.8)$ in all these examples. 

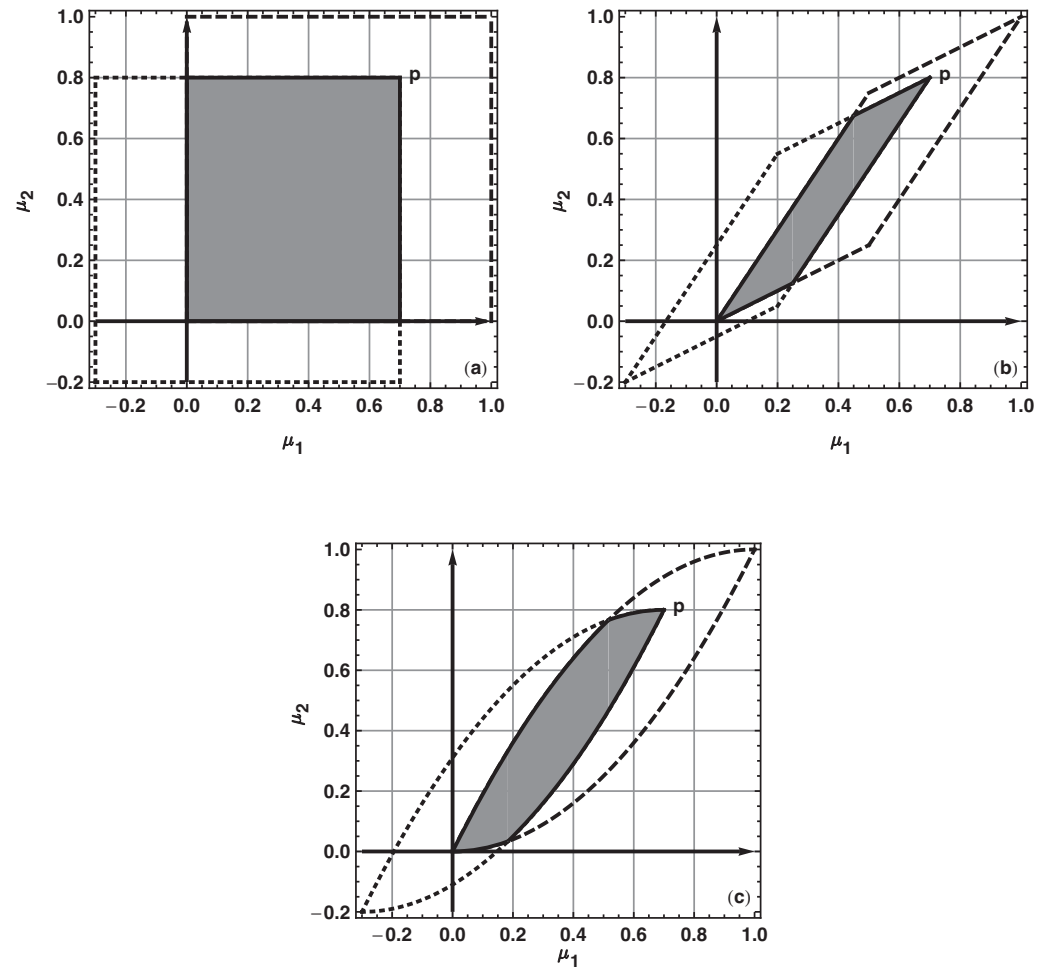

Figure 1. Plots (a)-(c) present the maximal subsets for the vector measures described in Examples 5.15.3, respectively, with $p=(0.7,0.8)$. The area enclosed by the dashed lines is the range $R_{\mu}(X)$. The area enclosed by the dotted lines is obtained by parallelly shifting the dashed area by $(-0.3,-0.2)$. The shaded area is formed by the intersection of the above two areas and represents the identical sets $R_{\mu}\left(Z^{*}\right), R_{\mu}^{p}(X)$ and $Q_{\mu}^{p}(X)$.

Example 5.1. Let $\mu_{1}$ and $\mu_{2}$ be singular. Then the range $R_{\mu}(X)$ is the unit square enclosed by the dashed lines in Fig. 1(a). The shaded area denotes the identical sets $R_{\mu}\left(Z^{*}\right), R_{\mu}^{p}(X)$ and $Q_{\mu}^{p}(X)$ with $p=(0.7,0.8)$.

Example 5.2. Consider the vector measure $\mu(d x)=\left(\mu_{1}, \mu_{2}\right)(d x)=(1, f(x)) d x$, where

$$
f(x)= \begin{cases}\frac{1}{2} & \text { if } x \in\left[0, \frac{1}{2}\right) ; \\ \frac{3}{2} & \text { if } x \in\left[\frac{1}{2}, 1\right] .\end{cases}
$$

Then the range of $R_{\mu}(X)$ is the area enclosed by the dashed lines in Fig. 1(b). The shaded area denotes the three identical sets $R_{\mu}\left(Z^{*}\right), R_{\mu}^{p}(X)$ and $Q_{\mu}^{p}(X)$ with $p=(0.7,0.8)$.

Example 5.3. Let $\mu(d x)=\left(\mu_{1}, \mu_{2}\right)(d x)=(1,2 x) d x$. Then the range $R_{\mu}(X)$ is the area enclosed by the dashed lines in Fig. 1(c). The shaded area denotes the three identical sets $R_{\mu}\left(Z^{*}\right), R_{\mu}^{p}(X)$, and $Q_{\mu}^{p}(X)$ when $p=(0.7,0.8)$. 


\section{Proof of Theorem 2.5}

For any $B \subseteq A$, denote $p(B)=\sum_{a \in B} p^{a}$, where either $A=\{1,2, \ldots\}$ or $A=$ $\{1, \ldots, n\}$ for some $n=1,2, \ldots$.

Lemma 6.1. Let $\mu=\left(\mu_{1}, \mu_{2}\right)$ be a two-dimensional finite atomless measure. If $p(B) \in R_{\mu}(X)$ for all $B \subset A$ and $\sum_{a \in A} p^{a}=\mu(X)$, then there exists a partition $\left\{Z^{a} \in \mathcal{F}: a \in A\right\}$ of $X$ such that $p^{a}=\mu\left(Z^{a}\right)$ for each $a \in A$.

Proof. Consider $p=\mu(X)-p^{1}$. According to Theorem 2.3, there exists a maximal subset $Z^{*} \in \mathcal{S}_{\mu}^{p}(X)$ and $R_{\mu}\left(Z^{*}\right)=Q_{\mu}^{p}(X)$. Let $Z^{1}=X \backslash Z^{*}, X^{1}=Z^{*}$, and $A^{1}=A \backslash\{1\}$. Note that $p^{1}=\mu\left(Z^{1}\right)$ and $p(B) \in R_{\mu}\left(X^{1}\right)$ for all $B \subseteq A^{1}$. Indeed, $p(B)+p^{1}=p(B \cup\{1\}) \in R_{\mu}(X)$. Thus, $p(B) \in R_{\mu}(X)-\{(\mu(X)-p)\}$, and in addition $p(B) \in R_{\mu}(X)$. Therefore, $p(B) \in Q_{\mu}^{p}(X)=R_{\mu}\left(X^{1}\right)$.

Now for $p^{2} \in\left\{p^{a}: a \in A^{1}\right\}$ there exists a maximal set $Z^{*} \in \mathcal{S}_{\mu}^{p}\left(X^{1}\right)$, where $p=\mu\left(X^{1}\right)-p^{2}$. Let $Z^{2}=X^{1} \backslash Z^{*}, X^{2}=Z^{*}$, and $A^{2}=A^{1} \backslash\{2\}$. Then $p^{2}=\mu\left(Z^{2}\right)$ and $p(B) \in R_{\mu}\left(X^{2}\right)$ for all $B \subseteq A^{2}$. The repetition of this procedure generates the desired partition $\left\{Z^{a} \in \mathcal{F}: a \in A\right\}$.

Proof of Theorem 2.5. The necessity is obvious. For the sufficiency, in view of Lemma 6.1, it is enough to prove that condition (ii) implies $p(B) \in R_{\mu}(X)$ for all $B \subseteq A$. If $B$ is finite, condition (ii) implies $p(B) \in R_{\mu}(X)$. If $B$ is infinite, let $B=$ $\left\{a^{1}, a^{2}, \ldots\right\}$ and $B_{n}=\left\{a^{1}, a^{2}, \ldots, a^{n}\right\}, n=1,2, \ldots$. Then $p(B)=\lim _{n \rightarrow \infty} p\left(B_{n}\right)$ and $p\left(B_{n}\right) \in R_{\mu}(X)$ for $n=1,2, \ldots$, according to condition (ii). Since $R_{\mu}(X)$ is closed, $p(B) \in R_{\mu}(X)$.

Finally, we show that when $m=2$, the Dvoretzky-Wald-Wolfowitz purification theorem for a countable image set $A$ [4, 6] is a particular case of Theorem 2.5. Let $p^{a}=\int_{X} \pi(a \mid x) \mu(d x), a \in A$. If these vectors $p^{a}$ satisfy conditions (i) and (ii) of Theorem 2.5. then Theorem 2.5] implies that the transition probability can be purified in the case of a countable $A$ and $m=2$. Indeed, for (i), obviously $\sum_{a \in A} p_{a}=\mu(X)$. For (ii), if $B \subseteq A$, then

$$
\sum_{a \in B} p^{a}=\sum_{a \in B} \int_{X} \pi(a \mid x) \mu(d x)=\int_{X} \pi(B \mid x) \mu(d x) \in R_{\mu}(X),
$$

where the inclusion follows from a version of Lyapunov's theorem [1, p. 218].

\section{REFERENCES}

1. J. R. Barra. Mathematical Basis of Statistics. Academic Press, New York, 1981. MR628863 (84h:62002)

2. A. Dvoretzky, A. Wald, and J. Wolfowitz. Elimination of randomization in certain problems of statistics and of the theory of games. Proc. Natl. Acad. Sci. 36(1950), 256-260. MR0035979 $(12: 40 \mathrm{c})$

3. A. Dvoretzky, A. Wald, and J. Wolfowitz. Elimination of randomization in certain statistical decision procedures and zero-sum two-person games. Ann. Math. Stat. 22(1951), 1-21. MR.0039228 (12:515a)

4. D. A. Edwards. On a theorem of Dvoretsky, Wald, and Wolfowitz concerning Liapounov measures. Glasg. Math. J. 29(1987), 205-220. MR901667 (88i:28017)

5. E. A. Feinberg and A. B. Piunovskiy. On strongly equivalent nonrandomized transition probabilities. Theory Probab. Appl. 54(2010), 300-307.

6. M. A. Khan and K. P. Rath. On games with incomplete information and the DvoretskyWald-Wolfowitz theorem with countable partitions. J. Math. Econom. 45(2009), 830-837. MR2564361 
7. P. Loeb and Y. Sun. Purification of measure-valued maps. Illinois J. Math. 50(2006), 747-762. MR 2247844 (2007j:28022)

8. A. A. Lyapunov. Sur les fonctions-vecteurs complètement additives. Izv. Akad. Nauk SSSR Ser. Mat. 4(1940), 465-478. MR0004080(2:315e)

9. Cz. Olech, The Lyapunov theorem: its extensions and applications, Lecture Notes in Math. 1446, Springer, 1990, 84-103. MR 1079760 (92b:49011)

10. D. A. Ross. An Elementary Proof of Lyapunov's Theorem. The Amer. Math. Monthly 112(2005), 651-653. MR.2158899 (2006e:28002)

11. R. Schneider. Convex Bodies: The Brunn-Minkowski Theory. Cambridge University Press, Cambridge, 2008. MR 1216521 (94d:52007)

Department of Applied Mathematics and Statistics, Stony Brook University, Stony Brook, New York 11794-3600

E-mail address: Peng.Dai@stonybrook.edu

Department of Applied Mathematics and Statistics, Stony Brook University, Stony Brook, New York 11794-3600

E-mail address: Eugene.Feinberg@sunysb.edu 